\title{
Enhancement for $\Phi$-OTDR Performance by Using Narrow Linewidth Light Source and Signal Processing
}

\author{
Meng ZHANG ${ }^{1}$, Song $\mathrm{WANG}^{2 *}$, Yuanwei $\mathrm{ZHENG}^{1}$, Yao YANG ${ }^{1}$, \\ Xingjie $\mathrm{SA}^{1}$, and $\mathrm{Li} \mathrm{ZHANG}^{2}$ \\ ${ }^{1}$ Guizhou Power Grid Information and Communication Company, Guizhou, 550003, China \\ ${ }^{2}$ Key Laboratory of Optical Fiber Sensing \& Communications (Ministry of Education), University of Electronic Science \\ and Technology of China, Chengdu, 611731, China \\ *Corresponding author: Song WANGＥ-mail: 394858749@qq.com
}

\begin{abstract}
In order to enhance the signal-to-noise-ratio of a distributed acoustic sensing system based on phase-sensitive optical time-domain reflectometry ( $\Phi$-OTDR), we have proposed a combination of segmented unwrapping algorithm, averaging estimation of phase difference, and infinite impulse response (IIR) filtering method. The enhancement of signal quality is numerically demonstrated. Moreover, we have studied the influence resulted from the light source noise on the $\Phi$-OTDR performance. The result has shown that when the linewidth of light source used in the $\Phi$-OTDR system is narrower, the performance of the system is better. In a word, such a $\Phi$-OTDR system could obtain higher quality demodulated signals when the narrower linewidth light source is chosen and the method of averaging estimation phase difference is used.
\end{abstract}

Keywords: Phase unwrap; frequency filter; phase noise

Citation: Meng ZHANG, Song WANG, Yuanwei ZHENG, Yao YANG, Xingjie SA, and Li ZHANG, "Enhancement for $\Phi$-OTDR Performance by Using Narrow Linewidth Light Source and Signal Processing,” Photonic Sensors, 2016, 6(1): 58-62.

\section{Introduction}

Phase-sensitive optical time-domain reflectometry ( $\Phi-O T D R)$ is a powerful technique that allows fully distributed vibration sensing with fast response and high sensitivity. It has been applied in many fields such as civil structure health monitoring and security guarding [1, 2]. Accurately, acquiring vibration location has been achieved in the reported $\Phi$-OTDR system by monitoring the amplitude change of backscattered light [3-5]. However, for some special applications such as seismic wave detection, it could not satisfy requirements without the ability of providing phase information. Recently, an I/Q phase demodulation technology using $90^{\circ}$ optical hybrid was proposed to achieve phase demodulation [6]. In order to improve the performance of $\mathrm{I} / \mathrm{Q}$ phase demodulation of the $\Phi-O T D R$, we have proposed the combination of segmented unwrapping algorithm, averaging estimation of phase difference, and infinite impulse response (IIR) filtering method. Further, we have studied the influence on the $\Phi-O T D R$ by introducing light source noise with zero mean and different variance.

\section{Schematic setup}

Compared with other $\Phi$-OTDR demodulation regimes, the most significant difference in our work is that a hybrid component is introduced to the system structure, as shown in Fig. 1 [6]. One of the

Received: 6 September 2015 / Revised: 16 November 2015

(C) The Author(s) 2015. This article is published with open access at Springerlink.com

DOI: $10.1007 / \mathrm{s} 13320-015-0283-7$

Article type: Regular 


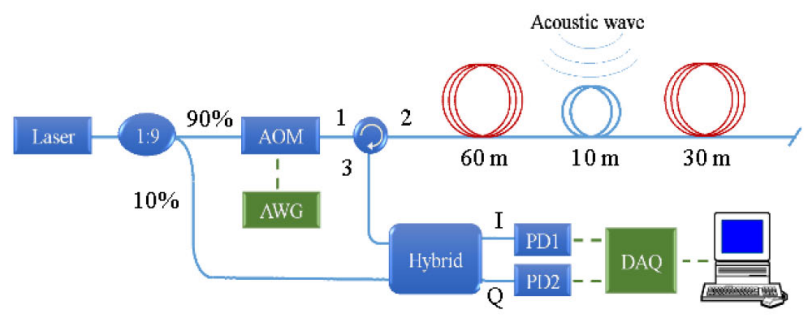

Fig. 1 Schematic setup.

hybrid outputs is the result of the interference between the local oscillator light and the backscattered light, which is called I channel. The other hybrid output called Q channel is the result of the interference between the $\pi / 2$ phase-shifted local oscillator and the backscattered light. In order to demodulate the information imposed on the optical fiber by simulation, it is necessary to obtain the arithmetic express of the wave of backscattered light [7]. When a coherent light pulse with a pulse width $W$ and an optical frequency $f$ is launched into the fiber at the time $t=k T$, where $k$ and $T$ stand for the numbers and the period of the light pulse, respectively, we obtain the wave of the backscattered light at the input end that is given by

$$
\begin{aligned}
e(t)= & \sum_{i=1}^{N} a_{i} \exp \left(-\alpha \frac{c \tau_{i}}{n_{f}}\right) \exp \left\{\mathrm { j } \left[2 \pi f\left(t-k T-\tau_{i}\right)\right.\right. \\
& \left.\left.+\theta\left(k T+\tau_{i}\right)\right]\right\} \operatorname{rect}\left(\frac{t-k T-\tau_{i}}{W}\right)
\end{aligned}
$$

where $a_{i}$ and $\tau_{i}$ are the amplitude and delay of the $i$ th backscattered wave, respectively, $N$ is the total scattering times of one light pulse, $\alpha$ is the fiber attenuation constant, $c$ is the velocity of light in a

$$
\begin{aligned}
\phi(y-x)= & \frac{2}{\operatorname{fix}\left(\frac{y-x+1}{2}\right)} \\
& \sum_{i=1}^{i=\mathrm{fix}\left(\frac{y-x+1}{2}\right)}\left\{\phi\left[y-\operatorname{fix}\left(\frac{y-x+1}{2}\right)+i\right]-\phi(x+i-1)\right\}
\end{aligned}
$$

where $y$ and $x(y>x)$ are not larger than $N$, and in the same group, the distance between $y$ and $x$ is not larger than the group length. In addition, the fix $(x)$ function is to get the integer part of $x$.

The third algorithm is to design a low-pass IIR filter with $8 \mathrm{kHz}$ stop-frequency to get the smooth vacuum, and the value of the rect function is one when $0<t-k T<W$, otherwise it will be zero. The delay $\tau_{i}$ corresponds to the distance $z_{i}$ from the input end to the $i$ th backscatter through the relation $\tau_{i}=2 n_{f} z_{i} / c$. The phase $\theta\left(k T+\tau_{i}\right)$ stands for the light source noise at the time $t=k T+\tau_{i}$, which obeys normal distribution with zero mean and variance $\sigma=2 \pi \tau \Delta v$ [8], where $\tau$ and $\Delta v$ are the period of the seed light pulse and the linewidth of the light source, respectively. With the help of the hybrid and the local oscillator, it is easy to get the light signals of I channel and $\mathrm{Q}$ channel, which are converted to analog electric signals by PD1 and PD2, respectively. Here, the analog electric signals are sampled with $50 \mathrm{MHz}$ sampling rate by the data acquisition (DAQ) card.

As for the segmented unwrapping algorithm, it means successively dividing the digital signals of I channel and Q channel of the traces into groups, i.e., every ten digital signals of I channel and Q channel as a group before unwrapping, which makes the phase $\phi(n)$ between $\mathrm{Q}$ channel and I channel continuous and beyond $[-\pi, \pi]$. In comparison with unwrapping all digital signals of I channel and Q channel, the segmented unwrapping algorithm limits the influence of the unwrapping error within the specific group and reduces the influence of total unwrapping errors. Another important algorithm is averaging estimation of phase difference, which operated after the segmented unwrapping algorithm. It is expressed as

\section{Simulation results and discussions}

In simulation, as shown in Fig. 1, we set the total fiber length as $100 \mathrm{~m}$, and only the fiber section between $60 \mathrm{~m}$ and $70 \mathrm{~m}$ bears external sine acoustic 
wave, which produces sinusoidal strain between $-200 \mathrm{n} \varepsilon$ and $+200 \mathrm{n} \varepsilon$ at $1500 \mathrm{~Hz}$, as shown in Fig. 2 . The repetition rate and width of light pulses are $50 \mathrm{kHz}$ and $100 \mathrm{~ns}$, respectively. The sampling rate of the DAQ card is configured as $50 \mathrm{MHz}$, in other words, the DAQ card could get the data of I channel and $\mathrm{Q}$ channel every two-meter length of the optical fiber, respectively.

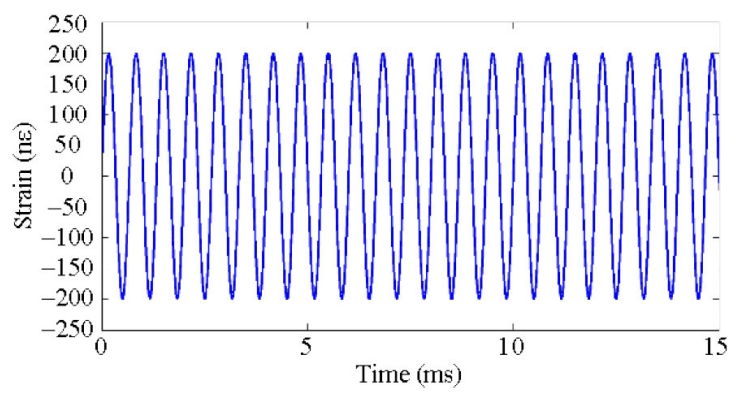

Fig. 2 Sinusoidal strain signal.

For the signal processing, specific implement of the segmented unwrapping algorithm is to obtain the caring data in the same group from the sampled data and then unwrap it. Here, the caring data, five digital data, are the data of the fiber between $60 \mathrm{~m}$ and $70 \mathrm{~m}$, but ten digital data of the fiber between $60 \mathrm{~m}$ and $80 \mathrm{~m}$ are in the same group, so the caring data in the same group are the data of the fiber between $60 \mathrm{~m}$ and $80 \mathrm{~m}$. In order to get phase difference of the unwrapped data of the fiber between $70 \mathrm{~m}$ and $60 \mathrm{~m}$, the direct phase difference of two points is the difference between the unwrapped data at $70 \mathrm{~m}$ and that at $60 \mathrm{~m}$. At the moment, the direct phase difference of two points is rough, and it becomes smooth when it passes the $8 \mathrm{kHz}$ low-pass IIR filter, as shown in Fig. 3(a). The direct smooth phase difference of two points in Fig. 3(a) is transformed by fast Fourier transform algorithm (FFT) to those in Fig. 3(b) when the spectrum of direct phase difference is needed to calculate the signal-tonoise-ratio (SNR) (the calculation method of SNR is reported in [9]). However, the averaging estimation smooth phase difference of two points is different from the direct smooth phase difference of two points, and the difference is the process of getting the phase difference. The process which is described as (2) has been done, and we could qualitatively find that the curve is smoother than that of direct phase difference in Fig.3(a). Moreover, the peak value of the spectrum of averaging estimation phase difference is about 2 times larger than that of the spectrum of direct phase difference. In order to quantitatively compare the quality of two methods [9], the method of averaging estimation phase difference and the method of direct phase difference, we calculate the SNRs of signals demodulated by them, and the result is that the SNR using the method of averaging estimation phase difference is $35.8 \mathrm{~dB}$, and the SNR using the method of the direct phase difference is $32.7 \mathrm{~dB}$. Obviously, the method of averaging estimation phase difference is better than the other demodulation method (in this situation, the linewidth of the light source is typical value $-100 \mathrm{~Hz}$ ).

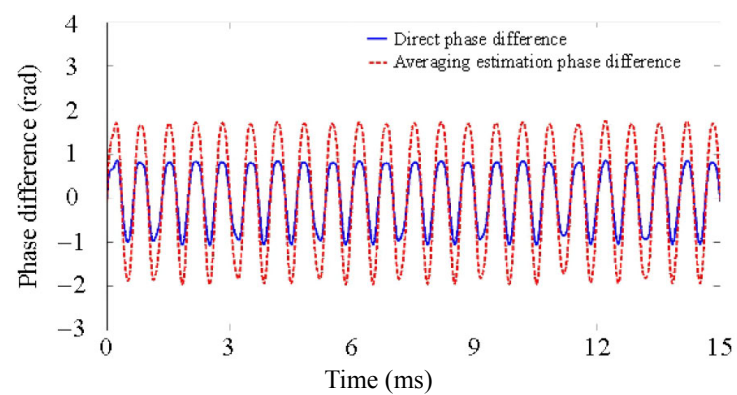

(a)

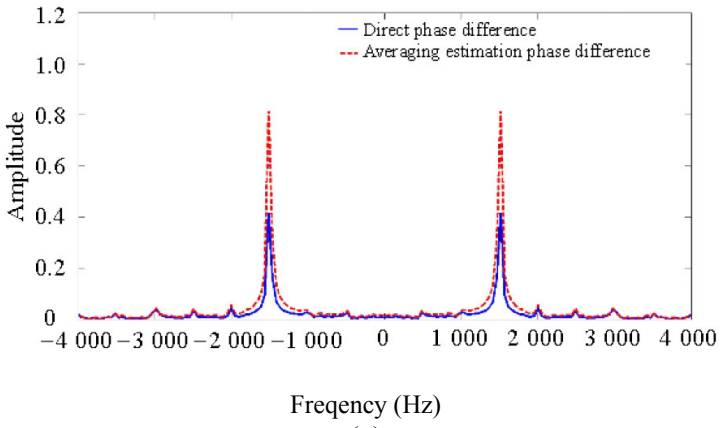

(a)

Fig. 3 Signals of the $\Phi$-OTDR system with the linewidth of $100 \mathrm{~Hz}$ light source: (a) demodulated signals and (b) spectra of demodulated signals.

Next, we discuss the effect that the different linewidths of light sources play on the demodulated 
signals using both the methods of averaging estimation phase difference and the direct phase difference. We set the linewidth as $100 \mathrm{~Hz}$ and $500 \mathrm{~Hz}$, and then we compare the phase differences in the each method, respectively. The results are presented in Fig. 4. It is easily found that the dot lines with $100 \mathrm{~Hz}$ linewidth are smoother and more perfectly fit the sine curve than the solid lines with $500 \mathrm{~Hz}$ linewidth, no matter which method is used. In order to accurately further study how the linewidth of the light source affects the performance of both methods, the SNRs of signals demodulated by both methods with different linewidths of the light source are calculated, as shown in Fig. 5. From Fig. 5, two characteristics are found, one is that the SNR descends when the light source linewidth increases, and the other characteristic is that the SNR of the demodulation signal using the method of averaging estimation phase difference is always larger than that of the demodulation signal using the method of the direct phase difference on the condition of the same linewidth of the light source. Besides, the SNR of the signal demodulated

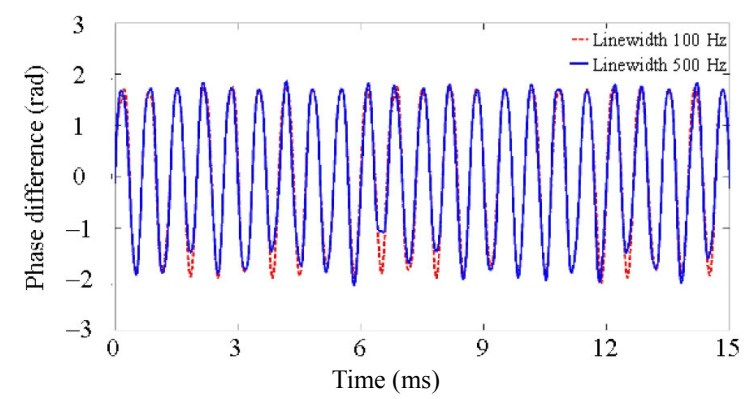

(a)

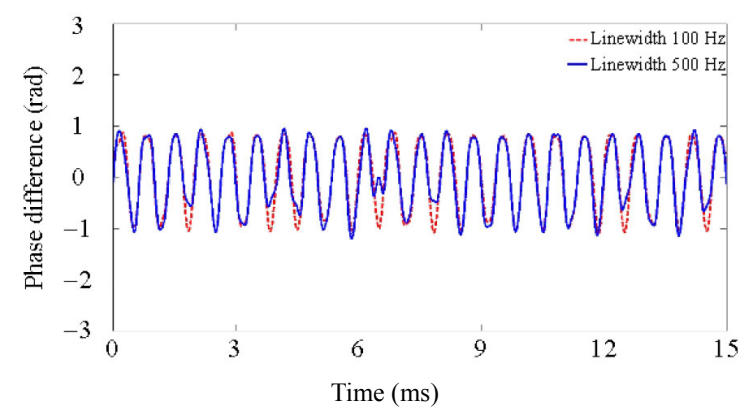

(b)

Fig. 4 Demodulated signals of using two methods: (a) the method of averaging estimation phase difference and (b) the method of direct phase difference. by the method of averaging estimation phase difference is up to $31.6 \mathrm{~dB}$ when the linewidth of the light source is $3500 \mathrm{~Hz}$. In a word, the SNR descends when the light source noise increases, however, the method of averaging estimation phase difference in performance is better than the other method.

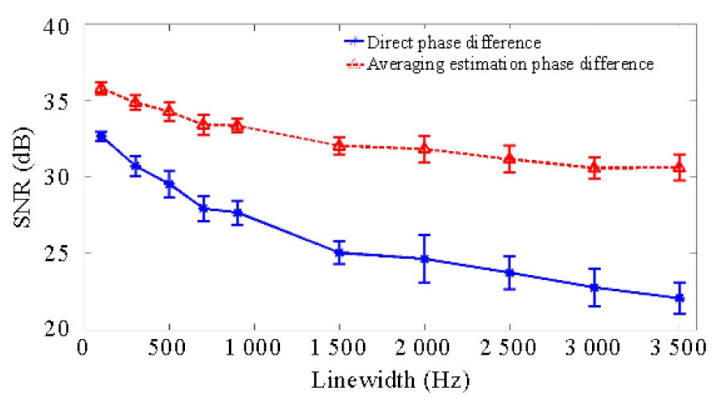

Fig. 5 SNRs of signals demodulated by two methods with different linewidths.

\section{Conclusions}

In order to enhance the performance of the distributed acoustic sensing system based on $\Phi-O T D R$, we have proposed the method of averaging estimation phase difference, which is proved better than the method of the direct phase difference by adding the light source with different linewidths (different level light source noises) to the $\Phi-O T D R$ system. Moreover, by comparing the SNRs of the demodulated signals of different linewidths of the light source, we find that demodulated signal is better when the linewidth of the chosen light source is narrower. Hence, a light source of a narrower linewidth is preferred to enhance the performance of the system.

In summary, the $\Phi$-OTDR system could obtain higher quality demodulated signals when we choose narrower linewidth light source and use the method of averaging estimation phase difference.

Open Access This article is distributed under the terms of the Creative Commons Attribution 4.0 International License (http:// creativecommons.org/licenses/by/4.0/), which permits unrestricted use, distribution, and reproduction in any medium, provided you give appropriate credit to the original author(s) and the source, 
provide a link to the Creative Commons license, and indicate if changes were made.

\section{References}

[1] H. Martins, S. Lopez, P. Corredera, M. Filograno, O. Frazao, and M. Herraez, "Coherent noise reduction in high visibility phase-sensitive optical time domain reflectometer for distributed sensing of ultrasonic waves," Journal of Lightwave Technology, 2013, 31(23): 3631-3673.

[2] J. C. Juarez, E. W. Maier, K. Choi, and H. F. Taylor, "Distributed fiber-optic intrusion sensor system," Journal of Lightwave Technology, 2005, 23(6): 2081-2087.

[3] Z. Wang, J. Zeng, J. Li, M. Fan, H. Wu, F. Peng, et al., "Ultra-long phase-sensitive OTDR with hybrid distributed amplification," Optics Letters, 2014, 39(20): 5866- 5869

[4] T. Zhu, X. Xiao, Q. He, and D. Diao, "Enhancement of SNR and spatial resolution in $\Phi-O T D R$ system by using two-dimensional edge detection method," Journal of Lightwave Technology, 2013, 31(17): 2851-2856.
[5] Z. Qin, L. Chen, and X. Bao, "Wavelet denoising method for improving detection performance of distributed vibration sensor," IEEE Photonics Technology Letters, 2012, 24(7): 542-544.

[6] L. Zhang, Z. Wang, S .Wang, N. Xue, X. Qian, M. Fan, et al., "Phase-sensitive optical time-domain reflectometry with I/Q demodulation," in Asia Communications and Photonics Conference 2015, Hong Kong, pp. AM1I.3, 2015.

[7] Y. Koyamad, M. Imahama, K. Kubota, and K. Hogari, "Fiber-optic distributed strain and temperature sensing with very high measurand resolution over long range using coherent OTDR," Journal of Lightwave Technology, 2009, 27(9): 1142-1146.

[8] K. Liang, Z. Pan, J. Zhou, Q. Ye, H. Cai, and R. Qu, "Multi-paramenter vibration detection system based on phase sensitive optical time domain reflectomer," Chinese Journal of Lasers, 2012, 39(8): 080004-1-080004-5.

[9] Z. Pan, K. Liang, Q. Ye, H. Cai, R. Qu, and Z. Fang, "Phase-sensitive OTDR system based on digital coherent detection," in Asia Communications and Photonics Conference 2011, Shanghai, pp. 83110S-1-83110S-6, 2011. 